\title{
Detección de poblaciones tóxicas de Microcystis spp. con distintas preferencias ambientales. Estudio de caso: embalse de Salto Grande
}

\section{Detection of toxic Microcystis spp. genotypes with different environmental preferences. Case study: Salto Grande reservoir}

Lepillanca, Facundo (1); Martínez de la Escalera, Gabriela (1); Bordet, Facundo (2); O’Farrell, Inés (3); Piccini, Claudia (1) (1) Departamento de Microbiología, Instituto de Investigaciones Biológicas Clemente Estable, IIBCE, Montevideo, Uruguay.

(2) Área de Gestión Ambiental, Comisión Técnica Mixta de Salto Grande, Montevideo, Uruguay.

(3) Departamento de Ecología, Genética y Evolución, Instituto IEGEBA (CONICET-UBA), Facultad de Ciencias Exactas y Naturales, Universidad de Buenos Aires, Buenos Aires, Argentina.

Contacto: claudia.piccini@gmail.com

RECIBIDO: 9/7/2018 -APROBADO: 11/10/2018

\begin{abstract}
Resumen
En este trabajo se evaluó la dinámica y toxicidad de Microcystis spp. en el embalse de Salto Grande en invierno y verano de 2013, así como la diversidad genética de sus poblaciones tóxicas mediante análisis de melting de alta resolución de amplicones del gen $m c y$ J. La abundancia de células de Microcystis spp. varió entre los distintos sitios y entre estaciones; en verano se detectó una abundancia significativamente mayor de células y genotipos tóxicos así como de microcistina-LR. Además, se detectaron dos grupos de genotipos mcyJ (clusters 1 y 2 ) con diferentes preferencias ambientales. El 1 estuvo integrado por muestras de Microcystis spp. obtenidas en condiciones de bajas temperaturas $\left(\leq 17,6^{\circ} \mathrm{C}\right)$ y mayor conductividad $\left(\sim 52-58 \mu \mathrm{S} \mathrm{cm}^{-1}\right)$, con bajo número de células y ausencia de microcistina-LR. Las muestras incluidas en el cluster 2 se caracterizaron por ser de verano (temperatura entre 17,6 y $\left.25,9^{\circ} \mathrm{C}\right)$, tener menor conductividad $\left(\sim 50-52 \mu \mathrm{S} \mathrm{cm}^{-1}\right)$, alta abundancia de células y microcistina-LR. Nuestros resultados sugieren que las poblaciones tóxicas de Microcystis que florecen en verano serían diferentes a las que se mantienen durante el invierno y que la temperatura y conductividad del agua serían variables relevantes que controlarían su abundancia y toxicidad.

Palabras clave: Microcystis, genotipos tóxicos, mcyJ, HRM, embalse Salto Grande.
\end{abstract}

\begin{abstract}
In this work, the dynamics and toxicity of Microcystis spp. in Salto Grande reservoir (Uruguay river), as well as the genetic diversity of their toxic populations were assessed in winter and summer during 2013. Cell abundance of Microcystis spp. varied amongst different sites and between seasons, showing a significantly higher cell and toxic genotype abundances (copies of $m c y E$ gene) and microcystin-LR concentration during summer. Furthermore, when genetic diversity of toxic Microcystis was analyzed by high resolution melting analysis of $m c y J$ amplicons, two genotypes exhibiting different environmental preferences were detected (cluster 1 and 2). Cluster 1 included samples found at water conditions of low temperature (lower than $17,6{ }^{\circ} \mathrm{C}$ ) and slightly higher conductivity $\left(\sim 52\right.$ to $\left.58 \mu \mathrm{S} \mathrm{cm}^{-1}\right)$, showing small Microcystis abundance and absence of microcystin-LR, whereas cluster 2 comprised samples from higher temperatures (between 17,6 and $25,9^{\circ} \mathrm{C}$ ) and lower conductivity $\left(\sim 50\right.$ to $\left.52 \mu \mathrm{S} \mathrm{cm}^{-1}\right)$ conditions, and with a higher cell abundance and microcystin-LR production. Our results suggest that toxic Microcystis spp. populations that bloom during summer are different from those that persist during winter and pointed to water temperature and conductivity as relevant variables that control their abundance and toxicity. Keywords: Microcystis, toxic genotypes, mcyJ, HRM, Salto Grande reservoir.
\end{abstract}

\section{Introducción}

Las floraciones son eventos en los que se produce un incremento de la biomasa de una o unas pocas especies de cianobacterias en períodos cortos de tiempo. La importancia de estos eventos radica en el impacto que tienen sobre el ecosistema acuático, ya que alteran el ciclado de los nutrientes y disminuyen la disponibilidad de luz y oxígeno, 
causando una disminución de la biodiversidad y deterioro de la calidad del agua (Sivonen y Jones, 1999; Havens, 2007; Karjalainen, et al., 2007). En Uruguay se han registrado floraciones de cianobacterias en diferentes ecosistemas acuáticos desde la década de los 80 (Quirós y Luchini, 1982; Feola, et al., 2008; Kruk, et al., 2015; Bonilla, et al., 2015) y las especies de cianobacterias formadoras de floraciones más frecuentes son Microcystis aeruginosa, Nodularia spumigena, Planktothrix agardhii y Cylindospermopsis raciborskii (Aubriot, et al., 2011; UNESCO, 2009; Vidal y Kruk, 2008; De León y Yunes, 2001; Bonilla, 1997).

Las floraciones son favorecidas por las actividades antropogénicas (actividad agrícola-ganadera, industrial y urbanización), tales como el uso de fertilizantes, el vertido al agua de desechos domiciliarios no procesados, entre otros (Mazzeo, et al., 2002; Chalar, 2009; Rodríguez-Gallego, 2010). Estas actividades aportan nutrientes (principalmente nitrógeno y fósforo) al ecosistema acuático y pueden causar su eutrofización, favoreciendo el crecimiento de cianobacterias (De León y Yunes, 2001). Una de las preocupaciones asociadas a la presencia de floraciones de cianobacterias es debida a la capacidad de producir toxinas (cianotoxinas) que tienen algunas especies. Entre las especies potencialmente tóxicas que producen floraciones en todo el mundo se encuentran las pertenecientes al género Microcystis (Huisman, et al., 2005). Estas tienen la capacidad de producir microcistinas, una cianotoxina ampliamente conocida y reportada (Dittmann y Wiegand, 2006). A pesar de que existe abundante información acerca de la ecología de Microcystis spp. en diversos ecosistemas, no hay un consenso acerca de las condiciones ambientales que favorecen la producción de microcistinas y la proliferación de poblaciones capaces de producirlas.

Se ha descrito que durante una floración de Microcystis spp. conviven poblaciones tóxicas y no tóxicas y que la presencia de los genes involucrados en la síntesis de microcistinas es el factor determinante de su toxicidad (Rinta-Kanto, et al., 2009). Así, las cianobacterias tóxicas serán portadoras de genes $m c y$ (genotipo tóxico, mcy+) y las no tóxicas serán aquellas que carecen de dichos genes (genotipo no tóxico, mcy-). La biosíntesis de microcistinas es realizada a partir de dos complejos enzimáticos conocidos como el complejo polikétido sintetasa (KPS) y el complejo péptido sintetasa (NRPS), cuyos genes se organizan en un cluster genético integrado por dos operones bidireccionales conocido como cluster $m c y$ ( $m c y A-J)$ (Tillett, et al., 2000).

Los primeros registros de floraciones de Microcystis spp. en Uruguay fueron en el embalse de Salto Grande (Quirós y Luchini, 1982) y en la actualidad se siguen registrando floraciones en todas las estaciones del año (Bordet, et al., 2017). Asimismo, Kruk et al. (2015) detectaron la presencia de floraciones de especies de Microcystis en el Río Uruguay tanto en verano como en invierno, sugiriendo que dadas las condiciones nutricionales adecuadas estos organismos son capaces de proliferar a bajas temperaturas. Estudios anteriores de nuestro grupo de investigación mostraron que a lo largo del gradiente ambiental comprendido por el Río Uruguay y el Río de la Plata existen diferentes genotipos tóxicos de Microcystis spp. identificados de acuerdo a las variaciones en las secuencias nucleotídicas del gen $m c y J$ (Martínez de la Escalera, comunicación personal). Cada genotipo identificado se asoció a un conjunto determinado de condiciones ambientales, principalmente en relación a la temperatura, salinidad y turbidez. Este hallazgo llevó a proponer la existencia de ecotipos de Microcystis spp. adaptados a distintas condiciones ambientales (Martínez de la Escalera, comunicación personal). En este contexto, existirían distintas poblaciones de Microcystis adaptadas a condiciones ambientales claramente diferentes, tales como baja temperatura o alta conductividad, lo que evidencia la existencia de una gran diversidad intraespecífica que sería crucial para el éxito y proliferación de estos organismos.

Basados en estos antecedentes, la hipótesis de este trabajo propone que la estrategia que presentan las especies tóxicas del género Microcystis para prosperar en condiciones ambientales diversas de temperatura, salinidad, turbidez, disponibilidad de nutrientes, etcétera, involucra la existencia de alta diversidad a escala intraespecífica. Esta variabilidad incluye poblaciones con distinta capacidad para adaptarse a diversas combinaciones de las variables ambientales consignadas. Con el fin de contribuir a dilucidar esta hipótesis, el objetivo principal de este trabajo fue evaluar la diversidad genética de poblaciones tóxicas de Microcystis en el embalse de Salto Grande y conocer las condiciones ambientales en las que prosperan. La diversidad genética se evaluó mediante el análisis de melting de alta resolución (HRM) de amplicones del gen mcyJ. La elección de este gen estuvo basada en evidencias que indican que no sufre recombinación (a diferencia de $m c y A, B, C$ ) y por tanto su secuencia presenta un mayor grado de conservación que la del resto de los genes del cluster mcy. Estas características lo convierten en candidato para estudios filogenéticos (Tanabe, et al., 2004, 2009; Kim, et al., 2010).

La técnica de high resolution melting (HRM por su sigla en inglés) se basa en la amplificación de un gen determinado mediante PCR en tiempo real y permite detectar poliformismos de nucleótido único (SNPs por su sigla en inglés). Por tanto, fue empleada para detectar variaciones en las secuencias del gen $m c y J$ teniendo en cuenta que el perfil de melting de una muestra estará integrado por los perfiles de cada organismo portador del gen allí presente. Esto determina que al hablar del genotipo tóxico de una muestra nos referimos al conjunto de genotipos mcyJ que la componen, cuya variabilidad se refleja en su perfil de melting.

\section{Materiales y Métodos}

\section{Sitio de estudio y muestras}

El embalse de Salto Grande comprende una obra de represamiento del Río Uruguay de carácter binacional argentino-uruguayo. Fue creado para la generación de energía hidroeléctrica, además de su utilización con fines domésticos y sanitarios, navegación y riego. Se presenta como un ambiente con dos subambientes diferenciados (Figura 1): una zona central que abarca un $70 \%$ de la superficie total, con un tiempo medio de permanencia histórico a cota máxima de 0,031 años, y cinco brazos laterales de distintas características (Quirós y Cuch, 1982; Quirós y Luchini, 1982). De ellos, el brazo Gualeguaycito está ubicado en las proximidades de la represa de Salto Grande correspondiente a la margen argentina, abarca un $8,5 \%$ de la superficie total, es el área más crítica respecto a la calidad del agua del embalse, y es zona de actividades náuticas deportivas y recreativas. A su vez, en la margen izquierda (uruguaya), 
de condiciones morfométricas similares, se ubica el brazo Itapebí, que presenta diferencias limnológicas significativas con el brazo de margen derecha. El sitio Las Palmeras constituye una playa con condiciones tipo litoral respecto a los sitios canal, es de uso recreativo intenso, y presenta los registros máximos históricos de densidad y toxinas. El embalse se ubica pocos kilómetros aguas arriba de las ciudades de Concordia (Argentina) y Salto (Uruguay), $29^{\circ} 43^{\prime}$ y $31^{\circ}$ $12^{\prime}$ 'S y $57^{\circ} 06^{\prime}$ y $57^{\circ} 55^{\prime} \mathrm{O}$. Por su temperatura media anual mayor a $15^{\circ} \mathrm{C}$ es considerado un sistema cálido tropical (Salas y Martino, 1991). En los brazos del embalse se han registrado floraciones de cianobacterias Microcystis spp. y Dolichospermum spp. (Quiros y Lucchini, 1982).

Se obtuvieron 19 muestras de tres estaciones de muestreo (Figura 1): Gualeguaycito, Las Palmeras (únicamente muestreado en los meses de invierno) e Itapebí en seis meses del año 2013 (febrero, abril, junio, julio, agosto, setiembre). En cada estación se extrajeron tres muestras a diferentes profundidades: nivel sub-superficial $(0,2 \mathrm{~m})$, límite inferior de la capa fótica (2,5 a $3 \mathrm{~m})$ y una muestra a un metro de fondo. Todas las muestras se obtuvieron empleando una botella de Van Dorn.

En cada muestreo se midió conductividad $\left(\mu \mathrm{S} \mathrm{cm}^{-1}\right)$, $\mathrm{pH}$, oxígeno disuelto $\left(\mathrm{mg} \mathrm{L}^{-1}\right)$ y temperatura del agua $\left({ }^{\circ} \mathrm{C}\right)$ mediante una sonda multiparamétrica Hydrolab DS5. Además, se determinó la concentración de nutrientes, nitrógeno total (NT, mg L $\left.\mathrm{L}^{-1}\right)$ y fósforo total (PT, mg $\left.\mathrm{L}^{-1}\right)$, clorofila-a (mg L-1), abundancia de Microcystis spp. (células $\mathrm{mL}^{-1}$ ) y concentración de microcistina-LR $\left(\mu \mathrm{g} \mathrm{L}^{-1}\right)$.

La concentración de PT y NT fueron determinadas siguiendo las técnicas Standard Methods (American Public Health Association, et al., 2005).

Para determinar la concentración de clorofila- $a$, las muestras de agua se almacenaron en bidones de $2 \mathrm{~L}$ y se filtraron luego de 3-6 horas empleando filtros GF/C (Whatman) que se conservaron en oscuridad a $-20^{\circ} \mathrm{C}$. La extracción se realizó con acetona $90 \%$ y se aplicó el método espectrofotométrico tricromático (Lorenzen, 1967).

\section{Abundancia y toxicidad de Microcystis spp.}

La abundancia de células de Microcystis spp. se estimó mediante conteo en cámaras de sedimentación de 1 y $20 \mathrm{~mL}$ en microscopio invertido (método Utermöhl descrito en Hasle, 1978). La concentración de microcistina-LR en las muestras de agua se determinó por HPLC-DAD en el LATU tanto para agua potable como para agua bruta con base en la Norma ISO 20179 (International Organization for Standarization, 2005; Lawton, et al., 1994). El límite de detección del ensayo fue de $0,03 \mu \mathrm{g} \mathrm{L}^{-1}$.

\section{Extracción de ADN}

Las muestras obtenidas se filtraron a través de filtros de celulosa de $0,45 \mu \mathrm{m}$ de tamaño de poro y $13 \mathrm{~mm}$ de diámetro (Millipore) y los filtros se aplicaron al método descrito por Martínez de la Escalera et al. (2014) con modificaciones. Los filtros fueron colocados en tubos de lisis con una matriz de esferas de cerámica (diámetro 1,4 mm) con $800 \mu \mathrm{l}$ de buffer de extracción (composición del buffer de extracción: 100 $\mathrm{mM}$ Tris-HCL $\mathrm{pH}=8.0,100 \mathrm{mM}$ EDTA $\mathrm{pH}=8,0,100 \mathrm{mM}$ Na-Fosfato $\mathrm{pH}=8,0,1,5 \mathrm{M}$ NACL, 1\% CTAB). Luego se ho-

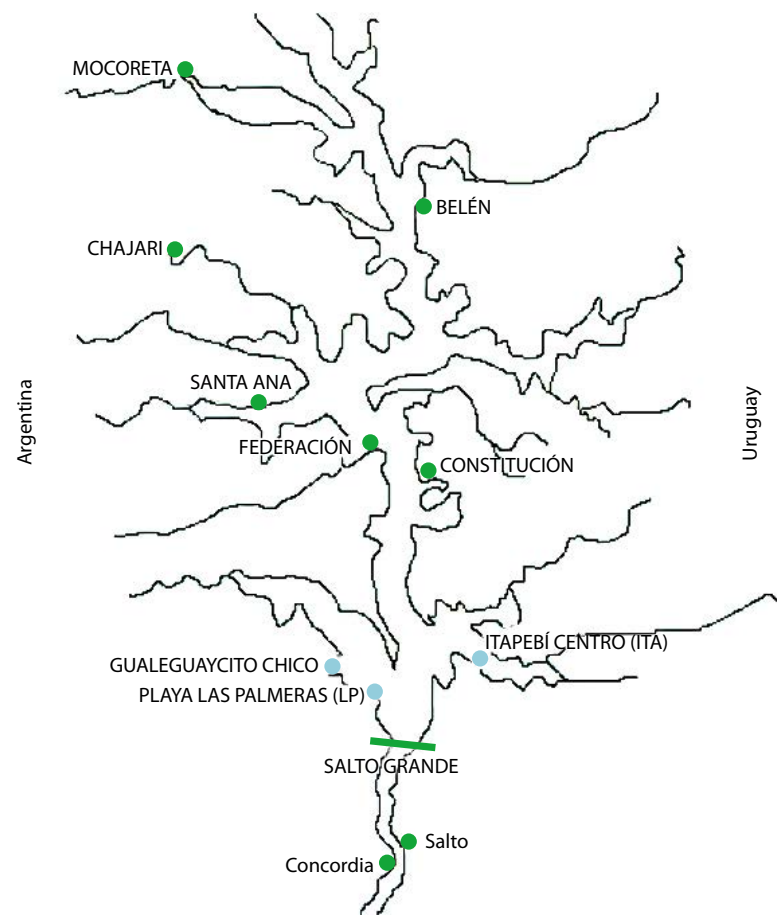

Figura 1. Embalse de Salto Grande. Los puntos azules indican los sitios de muestreo Gualeguaycito y Las Palmeras en Argentina e Itapebí en Uruguay. Los puntos verdes muestran las principales ciudades de la cuenca y la línea indica la localización de la represa de Salto Grande.

mogeneizó en el equipo Fast Prep (MP Biomedicals) durante 40 segundos a $6,0 \mathrm{~m} \mathrm{~s}^{-1}$. Posteriormente se continuó según el método de Martínez de la Escalera et al. (2014).

\section{PCR cuantitativo en tiempo real (qPCR)}

Se cuantificó la abundancia del gen $m c y E$ involucrado en la síntesis de la microcistina mediante qPCR utilizando cebadores específicos (Sipari, et al., 2010). En cada reacción se utilizó $10 \mathrm{nmol}$ de cada cebador, $2 \mu \mathrm{L}$ del ADN muestra (aproximadamente $5 \mathrm{ng}$ ) y $7,76 \mu \mathrm{L}$ del kit Power SYBR Green PCR (Invitrogen), con un volumen final de reacción de 20 $\mu \mathrm{L}$. La PCR constó de $2 \min$ a $50{ }^{\circ} \mathrm{C}, 15 \mathrm{~min}$ a $95^{\circ} \mathrm{C}$ y 40 ciclos de 15 s a $95^{\circ} \mathrm{C}, 30 \mathrm{~s}$ a $60^{\circ} \mathrm{C}$ y 30 s a $72{ }^{\circ} \mathrm{C}$ (Martínez de la Escalera, et al., 2017) y se realizó en un termociclador CFX96 Real Time System (BIORAD). Para cuantificar de manera absoluta el número de copias de $m c y E$ se empleó un clon y se realizaron diluciones seriadas de $1 / 1 \mathrm{E}-4$ a $1 / 1 \mathrm{E}-10$ para generar curvas de calibración y determinar el número de copias del gen por $\mathrm{mL}$ de muestra.

\section{Análisis de HRM (High Resolution Melting)}

Para realizar el análisis HRM primero se obtuvieron amplicones del gen $m c y J$ y luego se analizaron mediante la técnica de HRM. Del total de las muestras evaluadas en este trabajo (19) se emplearon 8 (3 muestras obtenidas en verano y 5 en invierno) que mostraron una amplificación eficiente del gen $m c y J$ mediante qPCR y por tanto fueron empleadas para realizar el HRM. Para ello, se utilizó el kit 
MeltDoctor HRM (Invitrogen) en un volumen final de reacción de $20 \mu \mathrm{L}$. Cada reacción contenía: $9.5 \mu \mathrm{L}$ de agua, $0,5 \mu \mathrm{L}$ de BSA (30 $\left.\mathrm{mg} \mathrm{mL}^{-1}\right), 7,76 \mu \mathrm{L}$ de mix MeltDoctor HRM, $0,12 \mu \mathrm{L}$ de cada cebador $(10 \mathrm{nmol})$ y $2 \mu \mathrm{L}$ del ADN muestra (aproximadamente $5 \mathrm{ng}$ ). Se utilizó el termociclador CFX96 Real Time System (BIORAD) y el software Bio-Rad Precision Melt Analysis.

\section{Análisis de datos}

Para detectar las relaciones entre las variables ambientales y biológicas se realizaron análisis de correlación de Spearman. El estudio de las diferencias significativas entre verano e invierno y entre los sitios de estudio en relación a los parámetros ambientales y biológicos fueron evaluados mediante el test de Kruskal-Wallis (KW).

Por último, los perfiles de melting obtenidos por HRM se analizaron mediante métodos de agrupamiento jerárquico aglomerativo: UPGMA (Unweighted Pair Group Method using Arithmetic averages), utilizando la distancia euclidiana. Los análisis estadísticos se realizaron empleando la plataforma libre R versión 3.4.1.

\section{Resultados}

\section{Caracterización ambiental}

La temperatura del agua mostró estacionalidad; la máxima temperatura se registró en verano $\left(25,9^{\circ} \mathrm{C}\right)$ y la mínima en invierno $\left(13,0^{\circ} \mathrm{C}\right)$. La conductividad del agua fue similar en ambas estaciones y el $\mathrm{pH}$ fue ligeramente alcalino. Respecto a las concentraciones de nutrientes (fósforo y nitrógeno total) no se observaron diferencias significativas entre verano e invierno y tampoco entre los sitios de muestreo (Tabla 1).
La concentración de clorofila $a$ fue significativamente mayor en verano en Gualeguaycito e Itapebí (KW, p < 0,05) (Figura 2A).

\section{Abundancia y toxicidad de Microcystis spp.}

En general, se encontró una abundancia de células de Microcystis spp. significativamente mayor en las muestras correspondientes al verano en comparación al invierno (KW, p < 0,05) (Figura 2B). En Gualeguaycito, la abundancia de Microcystis spp. varió entre 328 y 87.700 células $\mathrm{mL}^{-1}$. Por otro lado, Itapebí presentó una abundancia de Microcystis spp., que varió entre 585 y 267.000 células $\mathrm{mL}^{-1}$, mientras que Las Palmeras presentó menor abundancia de Microcystis spp. (0-6.975 células $\left.\mathrm{mL}^{-1}\right)$.

Cuando se analizó la abundancia de mcyE como proxy del potencial tóxico, el mayor valor se detectó en verano (5.228,3 copias $\mathrm{mL}^{-1}$ ). Sin embargo, durante el invierno también se detectó el gen $m c y E$ con una abundancia máxima de 2.490,9 copias $\mathrm{mL}^{-1}$. Si bien se observaron diferencias en la abundancia del gen $m c y E$ entre verano e invierno, estas no fueron significativas (KW, $\mathrm{p}<0,1)$.

$\mathrm{Al}$ evaluar la presencia y concentración de microcistina-LR, esta se detectó únicamente en verano (Gualeguaycito e Itapebí). En Gualeguaycito, la concentración de la toxina varió entre 5,5 y $96 \mu \mathrm{g} \mathrm{L}^{-1}$, mientras que en Itapebí se encontró entre 0 y 5,5 $\mu \mathrm{g} \mathrm{L} \mathrm{L}^{-1}$ (Figura 2). El número de copias del gen $m c y E$ se correlacionó positiva y significativamente con la temperatura del agua (Spearman, rho = $0,67, \mathrm{p}=0,01)$ y con la concentración de microcistina-LR (Spearman, rho $=0,45, p=0,049)$. Sin embargo, la correlación con la abundancia de células de Microcystis spp. fue no significativa para un p-valor de 0,05 (Spearman, rho $=$ $0,42 \mathrm{p}=0,07)$

\begin{tabular}{|c|c|c|c|c|c|}
\hline Sitios & $\begin{array}{c}\mathrm{T}\left({ }^{\circ} \mathrm{C}\right) \\
\text { media (rango) }\end{array}$ & $\begin{array}{c}\mathrm{K}\left(\mu \mathrm{S} \mathrm{cm} \mathrm{cm}^{-1}\right) \\
\text { media (rango) }\end{array}$ & $\begin{array}{c}\mathrm{pH} \\
\text { media (rango) }\end{array}$ & $\begin{array}{c}\mathrm{NT}\left(\mu \mathrm{g} \mathrm{L}^{-1}\right) \\
\text { media (rango) }\end{array}$ & $\begin{array}{c}\mathrm{PT}\left(\mu \mathrm{g} \mathrm{L}^{-1}\right) \\
\text { media (rango) }\end{array}$ \\
\hline Gualeguaycito invierno & $\begin{array}{c}14,6 \\
(13,0-17,0)\end{array}$ & $\begin{array}{c}52,7 \\
(48,3-54,6)\end{array}$ & $\begin{array}{c}7,8 \\
(7,53-8,27)\end{array}$ & $\begin{array}{c}900 \\
(500-1.100)\end{array}$ & $\begin{array}{c}40 \\
(60-20)\end{array}$ \\
\hline Gualeguaycito verano & $\begin{array}{c}23,4 \\
(21,1-25,9)\end{array}$ & $\begin{array}{c}52,1 \\
(49,3-57,4)\end{array}$ & $\begin{array}{c}8,5 \\
(7,49-9,42)\end{array}$ & $\begin{array}{c}750 \\
(500-900)\end{array}$ & $\begin{array}{c}60 \\
(40-80)\end{array}$ \\
\hline Itapebí invierno & $\begin{array}{c}15,1 \\
(13,8-17,0)\end{array}$ & $\begin{array}{c}58,4 \\
(54,7-60,5)\end{array}$ & $\begin{array}{c}7,6 \\
(7,45-7,78)\end{array}$ & $\begin{array}{c}770 \\
(500-1.000)\end{array}$ & $\begin{array}{c}40 \\
(20-70)\end{array}$ \\
\hline Itapebí verano & $\begin{array}{c}22,1 \\
(21,4-22,6)\end{array}$ & $\begin{array}{c}50,4 \\
(49,4-51,5)\end{array}$ & $\begin{array}{c}7,8 \\
(7,53-8,34)\end{array}$ & $\begin{array}{c}833 \\
(600-1.900)\end{array}$ & $\begin{array}{c}110 \\
(110-120)\end{array}$ \\
\hline Las Palmeras invierno & $\begin{array}{c}16,2 \\
(16,9-17,6)\end{array}$ & $\begin{array}{c}57,7 \\
(55,3-60,0)\end{array}$ & $\begin{array}{c}7,8 \\
(7,7-7,94)\end{array}$ & $\begin{array}{c}800 \\
(600-1.200)\end{array}$ & $\begin{array}{c}56 \\
(20-80)\end{array}$ \\
\hline
\end{tabular}

Tabla 1. Variables ambientales y nutrientes totales en cada sitio de muestreo: Gualeguaycito, Itapebí y Las Palmeras. Se muestran los valores medios y sus desvíos estándar. T: Temperatura, K: Conductividad, pH, NT: Nitrógeno total y PT: Fósforo total. 

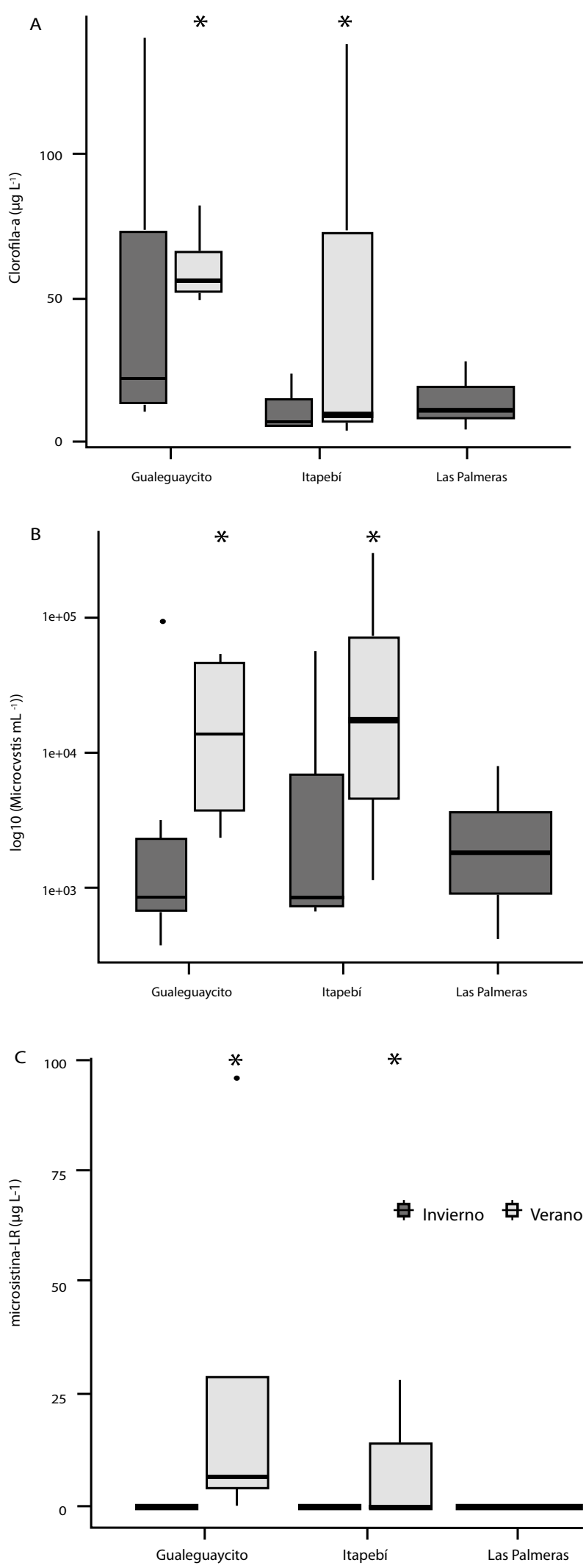

Figura 2. Variabilidad temporal (verano e invierno) y espacial (Gualeguaycito, Itapebí, Las Palmeras) de las

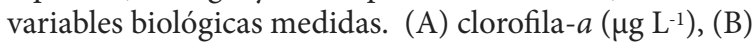
abundancia de Microcystis (células $\left.\mathrm{mL}^{-1}\right),(\mathrm{C})$ microcistina$\mathrm{LR}\left(\mu \mathrm{g} \mathrm{L}{ }^{-1}\right) .{ }^{*}$ diferencias significativas entre verano e invierno, test $\mathrm{KW} \mathrm{p}<0,05$.

\section{Diversidad estacional de genotipos mcyJ}

El análisis de cluster realizado a partir de los perfiles de melting de HRM del gen $m c y J$ obtenidos para cada muestra permitió distinguir dos grupos de genotipos tóxicos denominados cluster 1 y cluster 2 (Figura 3 ).
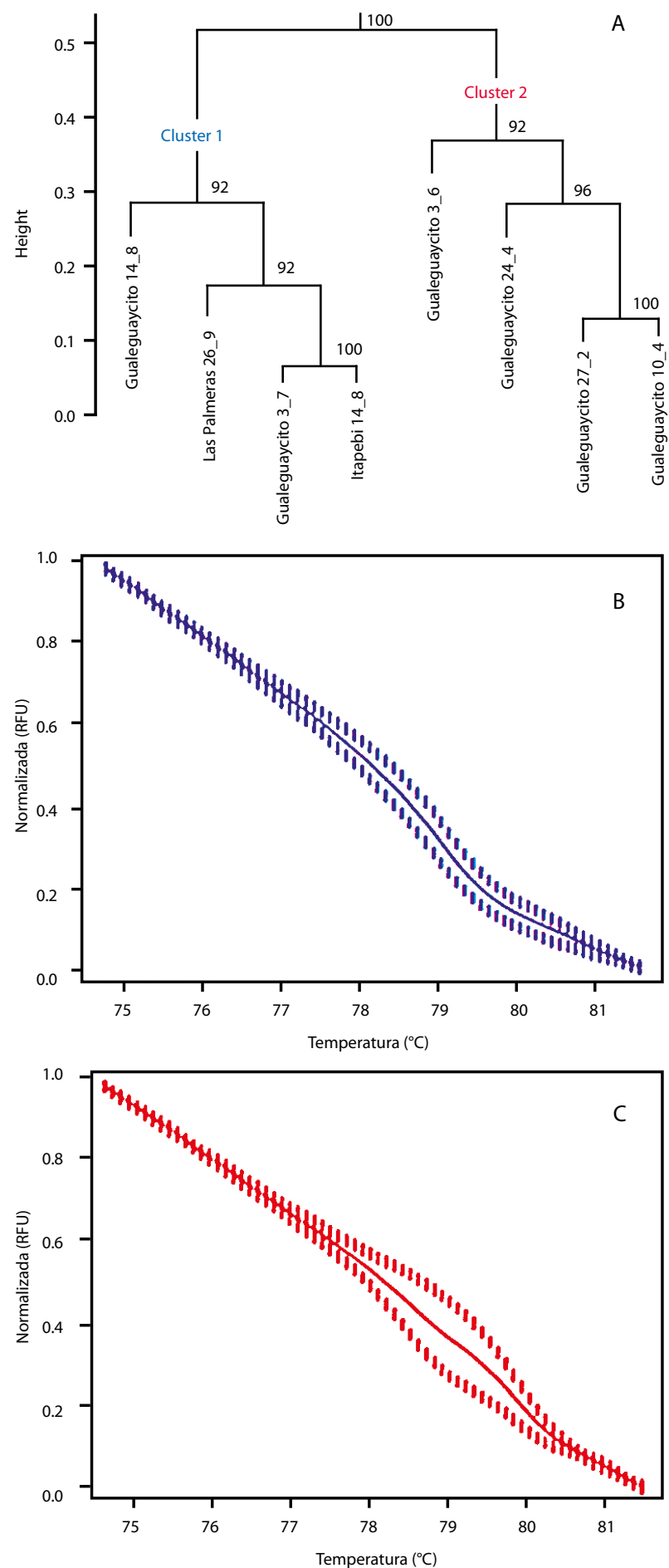

Figura 3. Dendrograma de los perfiles de melting agrupados utilizando la distancia euclidiana (A). Promedios (línea continua) y desvío estándar (líneas punteadas) de los perfiles de melting característicos del cluster 1 (B) y cluster 2 (C). 


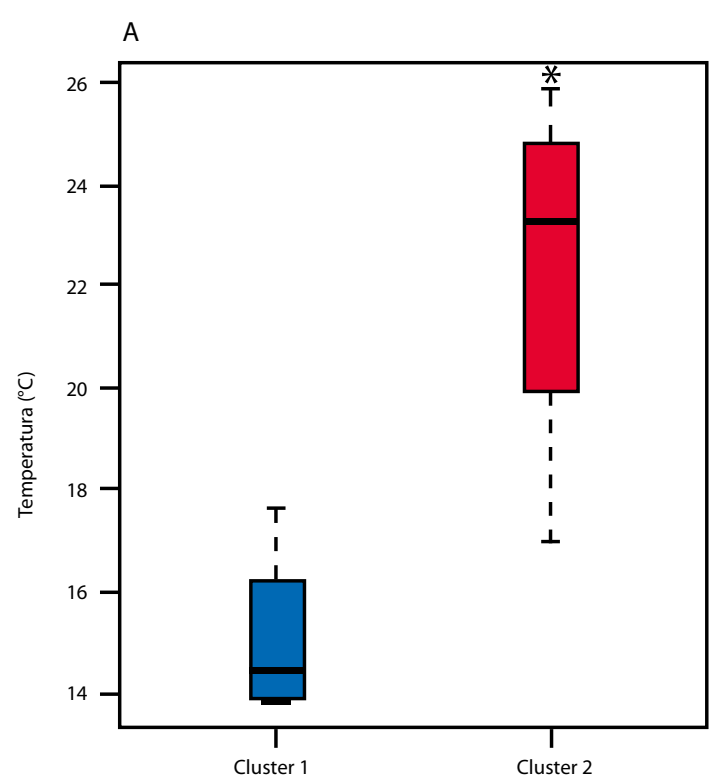

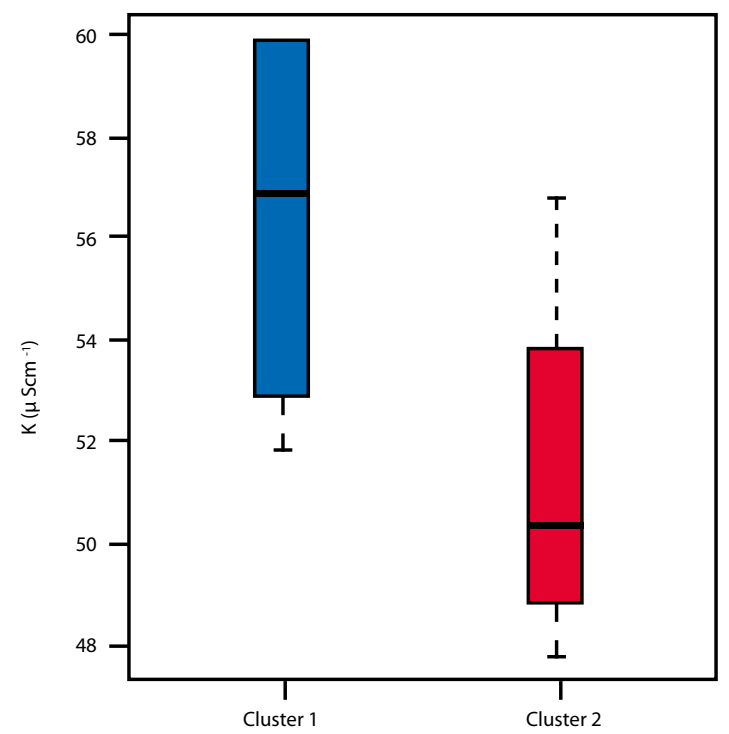

Figura 4. Variables ambientales asociadas a cada cluster. A) temperatura del agua $\left({ }^{\circ} \mathrm{C}\right)$ y $\left.\mathrm{B}\right)$ conductividad del agua $(\mathrm{K}, \mu \mathrm{S}$ $\mathrm{cm}-1) .{ }^{\star}$ Diferencias significativas entre ambos clusters, test de Kruskal-Wallis $(\mathrm{p}<0,05)$.
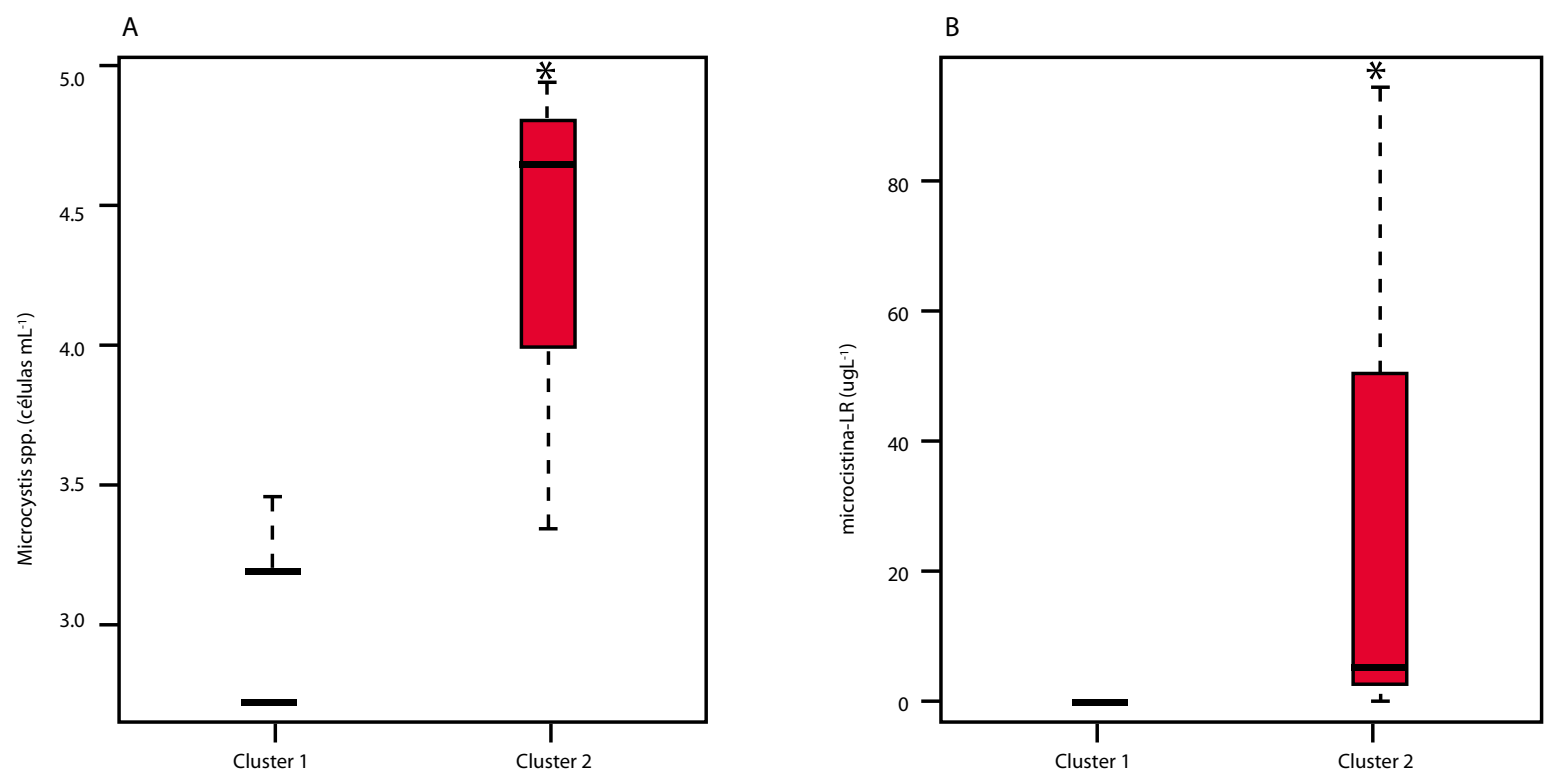

Figura 5. Abundancia de Microcystis sp. (células $\mathrm{mL}^{-1}$ ) (A) y concentración de microcistina-LR ( $\left.\mu g \mathrm{~L}^{-1}\right)(\mathrm{B})$ de las muestras en cada cluster. ${ }^{\star}$ Diferencias significativas entre ambos clusters, test de Kruskal-Wallis $(\mathrm{p}<0,05)$.

Se evaluaron las condiciones ambientales en las que aparecía cada cluster de genotipos y se observó que las muestras pertenecientes al cluster 1 aparecían a temperaturas bajas (entre 13,8 y $17,6^{\circ} \mathrm{C}$ ), mientras que el cluster 2 incluía muestras obtenidas en condiciones de temperatura entre 17,0 y $25,9{ }^{\circ} \mathrm{C}(\mathrm{KW}, \mathrm{p}<0,05)$ (Figura $\left.4 \mathrm{~A}\right)$. Asimismo, se observaron diferencias en la conductividad del agua entre ambos clusters aunque no fueron significativas (KW, $\mathrm{p}<$ $0,1)$. El cluster 1 estaba integrado por muestras cuya conductividad se encontraba en el rango entre 48 y $57 \mu \mathrm{S} \mathrm{cm}^{-1}$, mientras que las muestras que componían el cluster 2 pre- sentaron conductividades entre 52 y $60 \mu \mathrm{S} \mathrm{cm}^{-1}$ (Figura $4 \mathrm{~B}$ ). Asimismo, se observaron diferencias significativas en el número de células de Microcystis spp. entre ambos clusters (Figura 5A). El 1 presentó una abundancia de Microcystis spp. significativamente menor que el $2(0-2.875$ células $\left.\mathrm{mL}^{-1}\right)$, que presentó una variación entre 2.100 y 87.700 células $\mathrm{mL}^{-1}$. La toxicidad de las muestras (concentración de microcistina-LR) también presentó diferencias significativas entre clusters (KW, p < 0,05) (Figura 5B); el cluster 2 fue el único que presentó muestras tóxicas, con concentraciones de microcistina-LR de 5,5 a $96 \mu \mathrm{g} \mathrm{L} \mathrm{L}^{-1}$. 


\section{Discusión}

En este trabajo se evaluó la diversidad genética de Microcystis spp. en el embalse de Salto Grande mediante HRM del gen mcyJ. Con base en el análisis de los perfiles de melting de los amplicones obtenidos se pudo detectar la presencia de dos grupos de genotipos mcyJ (indicador de poblaciones potencialmente tóxicas), cada uno caracterizado por proliferar a distintas condiciones de temperatura y conductividad del agua (Figura 3). Asimismo, la mayor abundancia de células de Microcystis spp. y las mayores concentraciones de microcistina-LR se detectaron durante el verano, a temperaturas del agua de entre 21 y $23^{\circ} \mathrm{C}$. Sin embargo, no se encontró relación entre la abundancia y toxicidad de las cianobacterias con los nutrientes, ya que estos presentaron valores similares a lo largo del año.

Debido a la concentración de fósforo total, de clorofila- $a$ y a la abundancia de fitoplancton a lo largo del año, el embalse de Salto Grande se clasifica como un ambiente eutrófico (Chalar, et al., 1993) en el que las floraciones de cianobacterias son comunes (De León y Yunes, 2001; Chalar, 2009). Si bien la eutrofización es generalmente el factor que se considera responsable principal de las floraciones de cianobacterias (Reynolds, 2006; Schindler, et al., 2008), existe evidencia de que factores físicos tales como irradiancia, temperatura, turbulencia, mezcla vertical y flujo hidráulico contribuyen a la promoción de estos eventos (Paerl y Huisman, 2008; Kosten, et al., 2011; Paerl, 2014).

No obstante, hasta el momento no se conoce cuál es la combinación de condiciones ambientales e hidrológicas que estimulan la producción de toxinas por parte de las poblaciones tóxicas (O'Neil, et al., 2012; Pearl y Huisman, 2009). Desde los primeros estudios que analizaron la influencia del ambiente sobre la producción de microcistinas en cultivos, la temperatura ha sido propuesta como una variable relevante. En este sentido, se ha reportado que la producción de toxinas es generalmente mayor entre 20 y $25^{\circ} \mathrm{C}$ (van der Westhuizen y Eloff, 1985; Watanabe y Oishi, 1985; van der Westhuizen, et al., 1986). Más recientemente, Davis et al. (2009) observaron que al aumentar la temperatura en condiciones controladas que simulaban un ambiente eutrófico la tasa de crecimiento de células tóxicas de Microcystis (cuantificadas a través de la presencia del gen $m c y D$ ) aumentaba significativamente en relación a la de células no tóxicas. Incluso en algunos casos las células no tóxicas mostraban una disminución de sus tasas de crecimiento, lo que lleva a concluir que el incremento de la temperatura por encima de los valores promedio de los ecosistemas (por ejemplo en un escenario de aumento de temperatura por calentamiento global) llevaría a un aumento selectivo de las poblaciones tóxicas de Microcystis (Davis, et al., 2009; Joung, et al., 2011). En adición, otros estudios que involucran expresión génica mostraron que la fracción de células productoras de toxina en $M$. aeruginosa es significativamente mayor a temperaturas entre 20 y $26^{\circ} \mathrm{C}$ (Dziallas y Grossart, 2011). Estos resultados están en línea con los obtenidos en este trabajo, ya que se encontró mayor concentración de microcistina-LR a temperaturas cercanas a la óptima de crecimiento de las especies del género Microcystis $\left(21-23^{\circ} \mathrm{C}\right)$.

Diversos estudios han reportado resultados contrastantes al evaluar la asociación entre el ambiente y la diversidad de Microcystis spp. (Pobel, et al., 2012; Hu, et al., 2016), por lo que aún no hay evidencia clara acerca de cuáles son las variables ambientales que influyen sobre la diversidad ge- nética de Microcystis. Por ejemplo, basándose en el análisis del gen $m c y A$ mediante geles en gradiente desnaturalizante, Hu et al. (2016) encontraron que la variación genotípica de una comunidad de Microcystis se relacionaba con distintas variantes de microcistina, lo que implica que la composición de la comunidad determinaría el tipo de toxina producida. Asimismo, se ha reportado que a diferentes temperaturas $M$. aeruginosa sufre cambios significativos en el patrón de microcistinas que produce, mientras que el contenido total de toxina por célula no cambia (Amé y Wunderlin, 2005). Por tanto, la temperatura no solo estimularía el crecimiento selectivo de poblaciones tóxicas, sino también el tipo de microcistina producida por estos organismos.

Tanto los hallazgos reportados en la literatura como los de este trabajo sugieren que a temperaturas que optimizan la tasa de crecimiento la producción de toxinas sería máxima. Dado que el rol de la microcistina en la biología y ecología de los organismos que la producen aún no está esclarecido, la información generada hasta el momento (tanto a partir de cultivos como de muestras ambientales) en relación a la temperatura brinda nuevos enfoques para analizar este tema.

En relación a la conductividad y su efecto sobre Microcystis spp., al momento se ha reportado que su aumento no tendría impacto diferencial en poblaciones tóxicas y no tóxicas (Tonk, et al., 2007; Joung, et al., 2011; Tanabe, et al., 2018). Sin embargo, la mayoría de los estudios se centran en concentraciones de sales más altas que las encontradas normalmente en sistemas de agua dulce, evaluándose por lo general salinidades estuarinas a marinas. Cabe destacar que en este trabajo los cambios en la conductividad asociados a la aparición de uno u otro genotipo tóxico, si bien fueron significativos, estuvieron siempre en el rango de lo esperado para un sistema de agua dulce como el embalse de Salto Grande. Asimismo, dado que se evaluaron únicamente genotipos tóxicos, no es posible conocer el efecto de la conductividad sobre las poblaciones no tóxicas de Microcystis. Una hipótesis para explicar los resultados obtenidos es que el aumento de conductividad sea consecuencia de algún otro proceso hidrológico, por ejemplo la resuspensión de sedimento debida al viento (F. Bordet, comunicación personal), cuyo efecto sobre el metabolismo celular sea en detrimento de la producción de toxinas. Sería necesario realizar estudios específicos para detectar qué otra variable se asocia a los cambios de conductividad en el sistema de estudio.

Trabajos previos de nuestro grupo demostraron que la temperatura y la conductividad son las variables más relevantes para determinar la abundancia de genotipos tóxicos de Microcystis en el gradiente ambiental Río Uruguay-Río de la Plata (Kruk, et al., 2015; Martínez de la Escalera, et al., 2017). O'Farrell et al. (2012) propusieron que la intensidad y frecuencia de floraciones de cianobacterias en el embalse de Salto Grande podrían ser en gran medida explicadas por el ciclo hidrológico y por la morfología del embalse, encontrándose mayores acumulaciones de biomasa en los brazos donde desembocan cursos de agua tales como Gualeguaycito e Itapebí. Es en estos sitios que presentan baja profundidad, altos tiempos de residencia del agua y acumulación de nutrientes provenientes de la cuenca donde se generan floraciones masivas de Microcystis spp. tóxicas, probablemente asociadas a mayores temperaturas que las que alcanza el resto del embalse. En este contexto, el hallazgo de genotipos $m c y J$ de Microcystis spp. con diferente potencial tóxico y asociados a diferentes temperaturas sugiere que la variable es relevante 
no solo a la hora de condicionar la abundancia de poblaciones tóxicas, sino también para inducir la expresión de los genes involucrados en la síntesis de microcistinas.

Con base en los resultados obtenidos, es de esperar que aquellas condiciones ambientales que favorezcan altas temperaturas del agua en el embalse promuevan el crecimiento de poblaciones de Microcystis spp. con genotipo tóxico y estimulen la producción de toxinas.

\section{Reconocimientos}

Este trabajo fue realizado en el marco de la tesina para obtener el grado de Licenciado en Ciencias Biológicas (Facultad de Ciencias, UDELAR) de Facundo Lepillanca. Parcialmente financiado por PEDECIBA-Biología y por la Comisión Técnico-Mixta de Salto Grande.

\section{Referencias}

Amé, M.V. y Wunderlin, D.A., 2005. Effects of iron, ammonium and temperature on microcystin content by a natural concentrated Microcystis aeruginosa population. En: Water, Air, and Soil Pollution, 168, pp.235-248.

American Public Health Association, American Water Works Association y Water Environment Federation, 2005. Standard methods for the examination of water and wastewater. 21a ed. Washington: APHA. Standard Method 4500 P B - P E Ascorbic Acid Method and 4500 N.C Persulfate Method. Approved 1997-Rev. 2011.

Aubriot, L., Bonilla, S. y Falkner, G., 2011. Adaptive phosphate uptake behaviour of phytoplankton to environmental phosphate fluctuation. En: FEMS Microbiology Ecology, pp.1-16.

Bonilla, S., 1997. Composición y abundancia fitoplanctónica de tres embalses en cadena sobre el río Negro, Uruguay. En: Iheringia, Ser. Bot., 49, pp.47-61.

Bonilla, S., Haakonsson, S., Somma, A., Gravier, A., Britos, A., Vidal, L., De León, L., Brena, B., Pírez, M., Piccini, C., Martínez de la Escalera, G., Chalar, G., González-Piana, M., Martigani, F. y Aubriot, L., 2015. Cianobacterias y cianotoxinas en ecosistemas límnicos de Uruguay. En: INNOTEC, 10, pp.9-22.

Bordet, F., Fontanarrosa, M. y O'Farrell, I., 2017. Influence of light and mixing regime on bloom-forming phytoplankton in a subtropical reservoir. En: River Research and Applications, 33(8), pp.1315-1326. https://doi.org/10.1002/ rra.3189.

Chalar, G., De León, L., de León, R., Fabián, D. y Gorga, J., 1993. Evaluación de la eutrofización del embalse de Salto Grande. Análisis de las relaciones entre los parámetros

físico-químicos y biológicos. Informe final de la primera etapa. Montevideo: Sección Limnología, Facultad de Ciencias. (Informe inédito)

Chalar, G., 2009. The use of phytoplankton patterns of diversity for algal bloom management. En: Limnologica, 39(3), pp.200-208.

Davis, T.W., Berry, D.L., Boyer, G.L., Gobler, C.J, 2009. The effects of temperature and nutrients on the growth and dynamics of toxic and non-toxic strains of Microcystis during cyanobacteria blooms. En: Harmful Algae, 8(5), pp.715-725.
De León, L. y Yunes, J.S., 2001. First report of a Microcystincontaining bloom of the cianobacterium Microcystis aeruginosa in the La Plata River, South America. En: Environm. Toxicol., 16(1), pp.110-112.

Dittmann, E. y Wiegand, C., 2006. Cyanobacterial toxins occurrence, biosynthesis and impact on human affairs. En: Mol. Nutr. Food Res., 50, pp.7-17. doi:10.1002/ mnfr.200500162

Dziallas, C. y Grossart, H.P., 2011. Increasing oxygen radicals and water temperature select for toxic Microcystis sp. En: PLoS ONE, 6(9), pp.e25569.

Feola, G., Brena, B., Risso, J. y Sienra, D. y Echezarreta, M. E., 2008. Programa de monitoreo de agua de playas y costa de Montevideo. Informe temporada estival: 2007-2008 [En línea]. Montevideo: IMM. [Consulta: 12 de junio de 2017]. Disponible en: http://www.montevideo.gub.uy/sites/ default/files/informe_monitoreo_agua_playas_costas_ temporada_estival_2007-2008.pdf

Hasle, R.G., 1978. The inverted microscope method. En: Sournia, A., ed. Monographs on oceanographic methodology. Vol 6, Phytoplankton manual. Paris: UNESCO. pp. 88-96.

Havens, K.E., 2007. Cyanobacteria blooms: effects on aquatic ecosystems. En: Hudnell, K.H., ed. Cyanobacterial harmful algal blooms: state of the science and research. Vol. 619. New York: Springer: pp.675-732.

Hu, C., Rea, C., Yu, Z., Lee, J., 2016. Relative importance of Microcystis abundance and diversity in determining microcystin dynamics in Lake Erie coastal wetland and downstream beach water. En: Journal of Applied Microbiology, 120, pp.138-151. doi: 10.1111/jam.12983.

Huisman, J., Matthijs, H.C.P. y Visser, P.M., eds., 2005. Harmful Cyanobacteria. Amsterdam: Springer.

International Organization for Standarization, 2005. ISO 20179: Water quality. Determination of microcystins method using solid phase. Ginebra: ISO.

Joung, S.H., Oh, H.M., Ko, S.R., Ahn, C.Y., 2011. Correlations between environmental factors and toxic and nontoxic Microcystis dynamics during bloom in Daechung Reservoir, Korea. En: Harmful Algae, 10, pp.188-193.

Karjalainen, M., Engström-Öst, J., Korpinen, S., Peltonen, H., Pääkkönen, J-P. y Rönkkönen, S. et al., 2007. Ecosystem consequences of cyanobacteria in the Northern Baltic Sea. En: $A M B I O,(36)$, pp.195-202.

Kim, S.G., Joung, S.H., Ahn, C.Y., Ko, S.R., Boo, S.M., Oh, H.M., 2010. Annual variation of Microcystis genotypes and their potential toxicity in water and sediment from a eutrophic reservoir: population diversity of Microcystis spp. during algal bloom. En: FEMS Microbiol. Ecol., 74, pp.93-102.

Kosten, S., Huszar, V.M.L., Becarés, E., Costa, L.S., van Donk, E., Hansson, L.A., Jeppesen, E., Kruk, C., Lacerot, G., Mazzeo, N., de Meester, L., Moss, B., Lürling, M., Nõges, T., Romo, S. y Scheffer, M., 2011. Warmer climates boost cyanobacterial dominance in shallow lakes. En: Global Change Biology, 18, pp.118-126.

Kruk, C., Piccini, C., Segura, A., Nogueira, L., Carballo, C., Martinez de la Escalera Siri, G., Calliari, D., Ferrari, G., Simoens, M., Cea, J., Alcántara, I., Vico, P. y Miguez, D., 2015. Herramientas para el monitoreo y sistema de alerta de floraciones de cianobacterias nocivas: Río Uruguay y Río de la Plata. En: INNOTEC, 10, pp.23-39.

Lawton, L.A., Edwards, C. y Codd, G.A., 1994. Extraction and high-performance liquid chromatographic method for the 
determination of microcystins in raw and treated waters. En: Analyst, 119(7), pp.1525-1530.

Lorenzen, C.J., 1967. Determination of chlorophyll and pheopigments: spectrophotometric equations. En: Limnology and Oceanography, 12, pp.343-346.

Martínez de la Escalera, G., Antoniades, D., Bonilla, S., Piccini, C., 2014. Application of ancient DNA to the reconstruction of past microbial assemblages and for the detection of toxic cyanobacteria in subtropical freshwater ecosystems. En: Molecular Ecology, 23, pp.5791-5802.

Martínez de la Escalera, G., Kruk, C., Segura, A.M., Nogueira, L., Alcántara, I. y Piccini, C., 2017. Dynamics of toxic genotypes of Microcystis aeruginosa complex (MAC) through a wide freshwater to marine environmental gradient. En: Harmful Algae, 62, pp.73-83.

Mazzeo, N., Clemente, J., García-Rodríguez, F., Gorga, J., Kruk, C., Larrea, D., Meerhoff, M., Quintans, F., Rodríguez-Gallego, L. y Scasso, F., 2002. Eutrofización: causas, consecuencias y manejo. En: Domínguez, A. y Prieto, R.G., eds. Perfil ambiental del Uruguay. Montevideo: Nordan-Comunidad. pp.39-56.

O'Farrell, I., Bordet, F., Chaparro, G., 2012. Bloom forming cyanobacterial complexes co-occurring in a subtropical large reservoir: validation of dominant eco-strategies. En: Hydrobiologia, 698, pp.175-190.

O’Neil, J.M., Davis, T.W., Burford, M.A. y Gobler, C.J., 2012. The rise of harmful cyanobacteria blooms: The potential roles of eutrophication and climate change. En: Harmful Algae, 14, pp.313-334.

Paerl, H. y Huisman, J., 2008. Blooms like it hot. En: Science, 320, pp.57-58.

Paerl, H. y Huisman, J., 2009. Climate change: A catalyst for global expansion of harmful cyanobacterial blooms. En: Environ. Microbiol. Rep., 1, pp.27-37.

Paerl, H., 2014. Mitigating harmful cyanobacterial blooms in a human- and climatically-impacted world. En: Life, 4(4), pp.988.

Pobel, D., Godon, J.J., Humbert, J.F., Robin, J., 2012. Highfrequency monitoring of the genetic diversity and the potential toxicity of a Microcystis aeruginosa bloom in a French shallow lake. En: FEMS Microbiology Ecology, 79, pp.132-141. doi: 10.1111/j.1574-6941.2011.01203.x

Quirós, R. y Cuch, 1982. Características limnológicas del embalse de Salto Grande II: Distribución y Dinámica de nutrientes. Instituto Nacional de Investigación y Desarrollo Pesquero. En: Ecología Argentina, 8, pp.111-142.

Quirós, R. y Luchini, L., 1982. Características limnológicas del embalse de Salto Grande, III: Fitoplancton y su relación con parámetros ambientales. En: Rev. Asoc. Cienc. Nat. Litoral, 13, pp.49-66.

Reynolds, C.S., 2006. The ecology of freshwater phytoplankton. Cambridge: Cambridge University Press.

Rinta-Kanto, J.M., Konopko, E.A., DeBruyn, J.M., Bourbonniere, R.A., Boyer, G.L.y Wilhelm, S.W., 2009. Lake Erie Microcystis: relationship between microcystin production, dynamics of genotypes and environmental parameters in a large lake. En: Harmful Algae, 8, pp.665-673.

Rodríguez-Gallego, L., 2010. Eutrofización de las lagunas costeras de Uruguay: impacto y optimización de los usos del suelo. Montevideo: Facultad de Ciencias, UDELAR. (Tesis de Doctorado).
Salas, H. y Martino, P., 1991. A simplified phosphorous trophic state model for warm-water tropical state. En: Water Research, 25(3), pp.341-350.

Schindler, D.W., Hecky, R.E., Findlay, D. L., Stainton, M.P., Parker, B.R., Paterson, M.J., Beaty, K.G., Lyng, M. y Kasian, S.E.M., 2008. Eutrophication of lakes cannot be controlled by reducing nitrogen input: results of a 37-year whole-ecosystem experiment. En: Proceedings of the National Academy of Sciences, 32, pp.11254-11258.

Sipari, H., Rantala-Ylinen, A., Jokela, J., Oksanen, I., Sivonen, K., 2010. Development of a chip assay and quantitative PCR for detecting microcystin synthetase e gene expression. En: Appl. Environ. Microbiol., 76, pp.3797-3805.

Sivonen, K. y Jones, G., 1999. Cyanobacterial toxins. En: Chorus, I. y Bartram, J., eds. Toxic cyanobacteria in water: a guide to their public health consequences, monitoring and management. Londres: E \& FN Spon. pp.41-111.

Tanabe, Y., Kaya, K. y Watanabe, M.M., 2004. Evidence for recombination in the Microcystin Synthetase (mcy) genes of toxic cyanobacteria Microcystis spp. En: Journal of Molecular Evolution, 58, pp.633-641. doi: 10.1007/s00239-004-2583-1.

Tanabe, Y., Sano, T., Kasai, F. y Watanabe, M.M., 2009. Recombination, cryptic clades and neutral molecular divergence of the microcystin synthetase (mcy) genes of toxic cyanobacterium Microcystis aeruginosa. En: $B M C$ Evolutionary Biology, 9, pp.115. doi: 10.1186/1471-2148-9-115

Tanabe, Y., Hodoki, Y., Sano, T., Tada, K. y Watanabe, M.M., 2018. Adaptation of the freshwater bloom-forming cyanobacterium microcystis aeruginosa to brackish water is driven by recent horizontal transfer of sucrose genes. En: Frontiers in Microbiology, 9, pp.1150.

Tillett, D., Dittmann, E., Erhard, M., Von Dohren, H., Borner, T. y Neilan, B.A., 2000. Structural organization of microcystin biosynthesis in Microcystis aeroginosa PCC7806: an integrated peptide-polyketide synthetase system. En: Chemistry \& Biology, 7, pp.753-764.

Tonk, L., Bosch, K., Visser, P., M. y Huisman, J., 2007. Salt tolerance of the harmful cyanobacterium Microcystis aeruginosa. En: Aquatic Microbial Ecology, 46(2), pp.117-123.

UNESCO y Bonilla, Sylvia, ed., 2009. Cianobacterias planctónicas del Uruguay. Manual para la identificación y medidas de gestión. Montevideo: UNESCO. (Documento Técnico PHI-LAC, $\mathrm{N}^{\circ}$ 16)

van der Westhuizen, A.J. y Eloff, J.N., 1985. Effect of temperature and light on the toxicity and growth of the blue-green alga Microcystis aeruginosa (UV-006). En: Planta, 163(1), 55-59.

van der Westhuizen, A.J., Eloff J.N. y Krüger G.H.J., 1986. Effect of temperature and light (fluence rate) on the composition of the toxin of the cyanobacterium Microcystis Aeruginosa(UV-006). En: Arch Hydrobiol., 108, pp.145-154

Vidal, L. y Kruk, C., 2008. Cylindrospermopsis raciborskii (Cyanobacteria) extends its distribution to latitude 34 $53^{\prime}$ 'S: taxonomical and ecological features in Uruguayan eutrophic lakes. En: Pan-American Journal of Aquatic Sciences, 3, pp.142-151.

Watanabe, M.F. y Oishi, S., 1985. Effects of environmental factors on toxicity of a cyanobacterium (Microcystis aeruginosa) under culture conditions. En: Applied and Environmental Microbiology, 49(5), pp.1342-1344. 